Article

\title{
Research on Simplified Model of AC/DC Hybrid Microgrid for Fault Analysis
}

\author{
Tianliang Yao ${ }^{1}$, Zhiwei $\mathrm{Li}^{1}{ }^{1}$, Jiping $\mathrm{Qu}^{1}$, Zhaoxiong $\mathrm{Li}^{1}{ }^{1}$, Qi Zhao ${ }^{1}$ and Guopeng Zhao ${ }^{2, *}$ (I) \\ 1 Gansu Electric Power Design Institute Limited Company of China Energy Construction Group, \\ Lanzhou 730050, China; yaotianliang@163.com (T.Y.); gd050706@126.com (Z.L.); qujiping142@163.com (J.Q.); \\ lizhaoxiong889@126.com (Z.L.); petty02070627@sina.com (Q.Z.) \\ 2 School of electrical and electronic engineering, North China Electric Power University, Beijing 102206, China \\ * Correspondence: zhaoguopeng@ncepu.edu.cn
}

Received: 2 February 2020; Accepted: 17 February 2020; Published: 20 February 2020

\begin{abstract}
The AC/DC hybrid microgrid, which takes into account the access requirements of AC and DC sources and loads, optimizes the structure of traditional distribution networks. The application of power electronic transformers as the core of its energy management, with electrical isolation and accurate control of the voltage, current and power flow by the control system, enables the microgrid to achieve a more flexible and stable transmission mode. Because the power electronic transformer combines the power electronic device and the high-frequency transformer, its frequent switching causes the electromagnetic transient simulation to take too long. Therefore, by simplifying control loops and converters, this paper proposes a simplified model for the microgrid system power flow and the dynamic response under exposure to a fault. The mathematical model equivalent simplification method is used in this paper. This method is concise and efficient and does not rely on the performance of a computer or change the program algorithm of the software. The simplified model was built based on PSCAD (Power System Computer Aided Design) simulation software and was carried out under short circuit fault conditions to verify its validity. The comparison of the simulation's time consumption and accuracy shows that model simplification can significantly improve the simulation speed, with an acceptable error rate, and its dynamic response maintains good consistency with that of the detailed electromagnetic transient model. Therefore, it can be applied to the transient electromagnetic simulation fault analysis of the AC/DC hybrid microgrid.
\end{abstract}

Keywords: control loop; converter; electromagnetic transient model; microgrid; simplified model

\section{Introduction}

As a system with various distributed generators, AC and DC loads, and self-adjusting and control capabilities [1,2], the microgrid applies power electronic transformers, which not only enables it to carry out the power transmission and electrical isolation of traditional transformers, but also realizes harmonic and reactive power compensation. The microgrid supports accurate and bidirectional power flow regulations [3-5] and has broad application prospects in this field. In [3-5], the topology and control strategy of the AC/DC hybrid microgrid is proposed to achieve the reliable and efficient operation of the AC/DC hybrid microgrid. In [6], Power Electronics Transformer (PET) topologies for three-phase AC distribution networks were discussed. By using space vectors, a mathematical model for MMC and a method for controlling the power flow were proposed. In [7], the typical control strategy for parallel operation was studied to realize the rational distribution of active and reactive power between power electronics transformers operating in parallel. In [8], a new type of power distribution transformer for the power distribution system was proposed, called the flexible 
power distribution unit, which revealed the energy exchange mechanism between the power grid and the load.

The microgrid electromagnetic transient model can simulate the dynamic process of the system in detail, but with the high order and non-linearity of the system, the switching process of a large number of power electronic devices increases the computational complexity and time-consumption of the simulation. Since the electromagnetic transient model mainly studies the transient process response at the microsecond level, where the nonlinearity and action characteristics of the components are considered, for different research focuses, changing the response time scale of the simulation model can improve the simulation efficiency. In view of the research on the characteristics of the microgrid output, it is not necessary to pay attention to the dynamic response process of its internal power electronic devices, so simplifying the processing can significantly improve the simulation efficiency.

In [9], an improved d-q-axis Flexible Positive and Negative Sequence Control Method (FPNSC) was proposed to ensure the safety of converters and facilities in the AC grid with unbalanced voltages. The study presented in [10] provided a detailed analysis of cable failures in VSC-based DC networks and identified the most severe failure stages to avoid. In [11], line-to-ground fault transients were analyzed in different neutral ground configurations. In [12,13], the fault simulation of the microgrid was established, the fault characteristics of which were analyzed.

In [14], a reduced-order small-signal model for multi-port power electronic transformers that derives the reduced-order model by using the participation factors of the system pattern as state variables was proposed. However, the state-space average method and the eigenvalue analysis were used in the paper, which are relatively complicated.

In [15], a reduced-order small-signal model of a microgrid system capable of operating in grid-connected and islanded conditions whose nonlinear equations are derived in the d-q reference system and then linearized around a stable operating point, was proposed. However, due to the nonlinearity of the system, the linearization results lost some accuracy. In [16], an efficient and accurate reduced-order model for an inverter-based microgrid was proposed, where the influence of network dynamics can be properly considered. However, it is not suitable for research under fault conditions. In [17], a mathematical model of an island microgrid with a linear load and inverter under frequency and voltage drop control was proposed. This was established by introducing appropriate state-space transitions to maintain the stability consistent with that of the original model using singular perturbation techniques. In [18], the singular perturbation method was used to simplify a full-order model of a microgrid with three inverter-based distributed generation systems (DG). However, due to the introduction of higher-order matrices, the singular perturbation method it used is much more complicated than the mathematical model equivalent simplification method.

In [19], a reduced-order small-signal model for electromechanical analysis was proposed, where each micro source is modeled in its local state space form. In [20], a small-signal model of a two-terminal HVDC system was established using the modularization method, which compares the effects of predictive control and proportional integral control on system stability. Although this method can completely retain the modal information of the system, the complicated derivation process and calculation result in high order of the model.

In this paper, a simplified model of an AC/DC hybrid microgrid for fault analysis is proposed through the mathematical model equivalent simplification method to ensure the effectiveness of the control system and the observability of parameter variables. By simplification and order reduction, the microsecond electromagnetic transient simulation, the time scale of which is increased from 10 to $20 \mathrm{~ms}$, not only improves the simulation efficiency, but also ensures its accuracy.

\section{Methods}

The AC-DC hybrid microgrid includes various distributed generators, energy storage devices, control, and communication systems. A two-end power supply AC/DC hybrid microgrid structure is shown in Figure 1. 


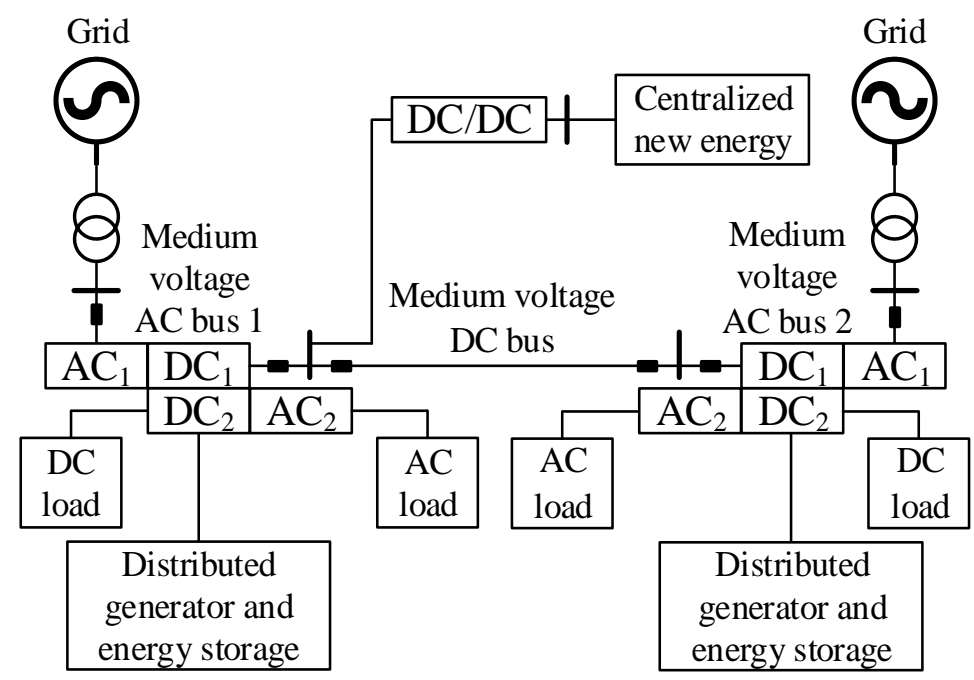

Figure 1. Two-end power supply AC/DC hybrid microgrid structure.

The AC-DC hybrid microgrid including three-stage AC-DC-AC power electronic transformers with $A C$ and DC interfaces improves the connection between the microgrid and the utility grid and various $\mathrm{AC}$ and DC sources and loads. The power electronic transformer consists of three parts: the input stage, isolation stage, and output stage. The input stage connected to the utility grid is a rectifying part, which rectifies the input alternating current at $50 \mathrm{~Hz}$ into direct current; the isolation stage consists of a pulse modulation, high frequency transformer, and pulse demodulation [9], which converts the DC inverter of the input stage output into a high frequency square wave and then couples it to the secondary side through a transformer and then rectifies to a direct current to supply power to the DC load; the output stage is the inverter part, which inverts the DC output of the isolation stage into a three-phase $50 \mathrm{~Hz}$ AC power supply for the load. Its structure and control system are shown in Figure 2.

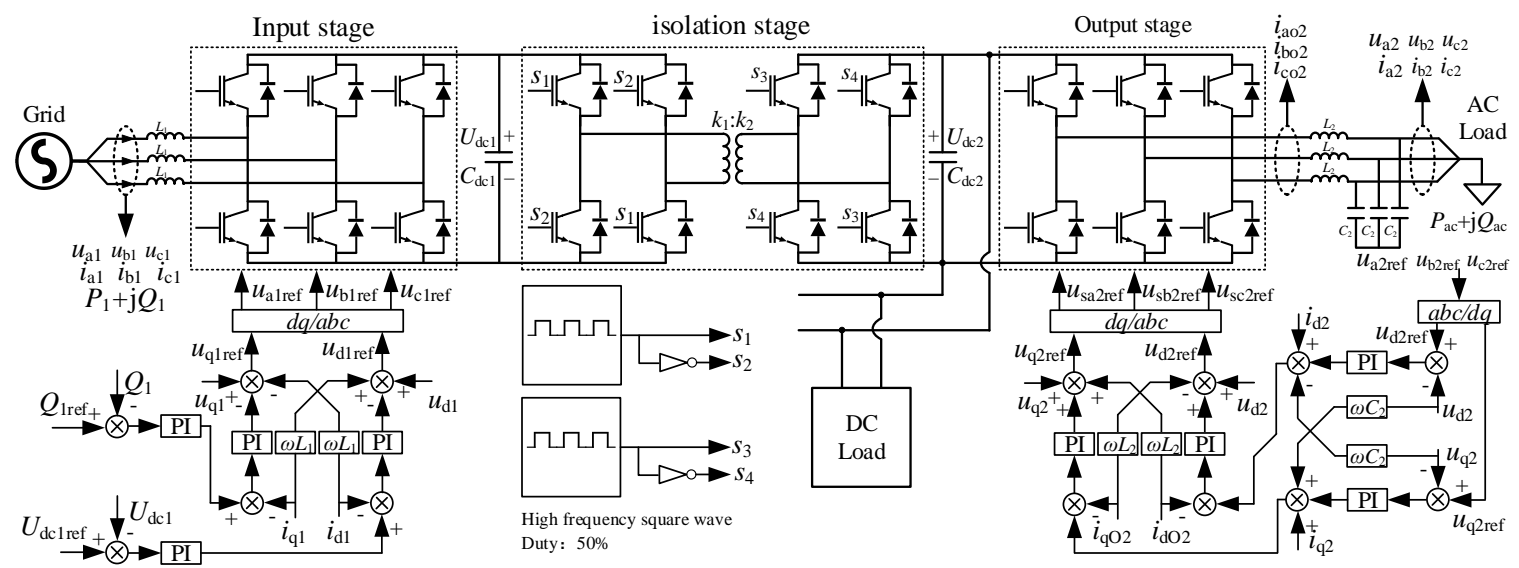

Figure 2. Detailed model structure of the microgrid.

Both the input and output stage control systems use dual loop control. The outer loop is the voltage and power loop, and the inner loop is the current loop. Current inner loop decoupling control is shown in Figure 3. 


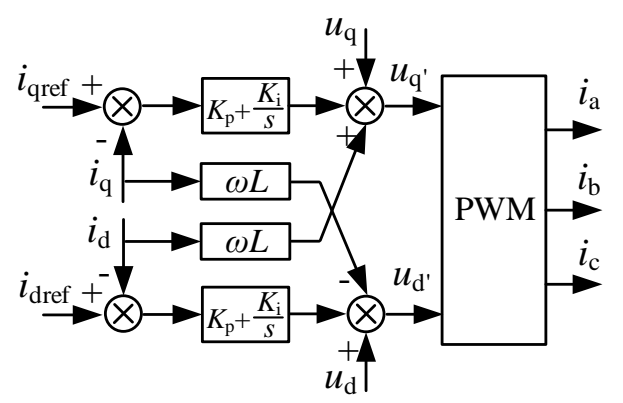

Figure 3. Current inner loop decoupling control.

Due to the symmetry of the two current inner loops, $i_{\mathrm{q}}$ is taken as the object of discussion. The delay of the current inner loop signal sampling and the small inertia characteristics of the Pulse Width Modulation (PWM) control are considered. The structure of the decoupled $i_{\mathrm{q}}$ current inner loop is shown in Figure 4.

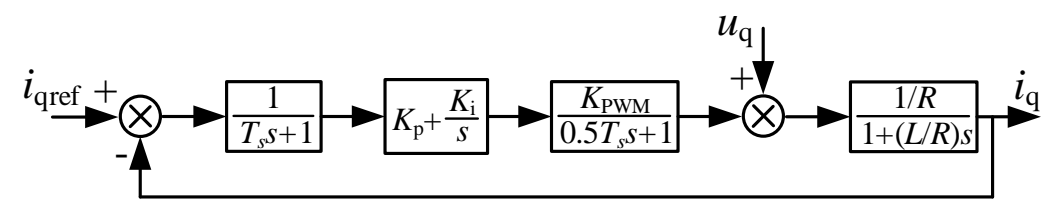

Figure 4. Current inner loop structure.

$T_{\mathrm{S}}$ is the sampling period of the current inner loop (which is also the PWM switching period), and $K_{\mathrm{PWM}}$ is the equivalent gain of the bridge PWM. To simplify the analysis, the perturbation of $u_{\mathrm{q}}$ is not considered, and the PI regulator transfer function is written as a pole-zero form, as shown in Equation (1).

$$
\left\{\begin{aligned}
K_{\mathrm{p}}+\frac{K_{\mathrm{i}}}{s} & =K_{\mathrm{p}} \frac{\tau s+1}{\tau s} \\
K_{\mathrm{i}} & =\frac{K_{\mathrm{p}}}{\tau}
\end{aligned}\right.
$$

The small time constants $0.5 T_{\mathrm{S}}$ and $T_{\mathrm{S}}$ are combined to produce a simplified current inner loop structure, as shown in Figure 5.

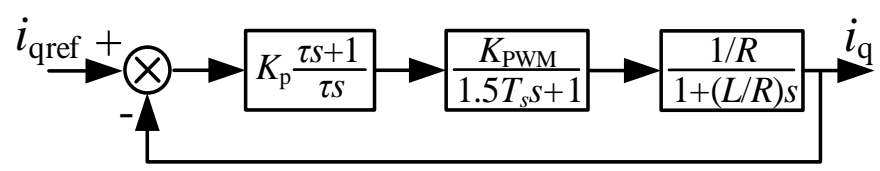

Figure 5. Simplified current inner loop structure.

Due to the high switching frequency of power electronics, the switching period is very short. The transfer function is approximately equivalent to 1 . Since the current inner loop responds faster than the outer loop, the response time scale is small. Therefore, the simplified model ignores the dynamic process of the current inner loop in the double loop control, where the input value of the inner loop is equal to the output value, and the converter is replaced with the controlled power sources. The simplified model structure and control system are shown in Figure 6. 


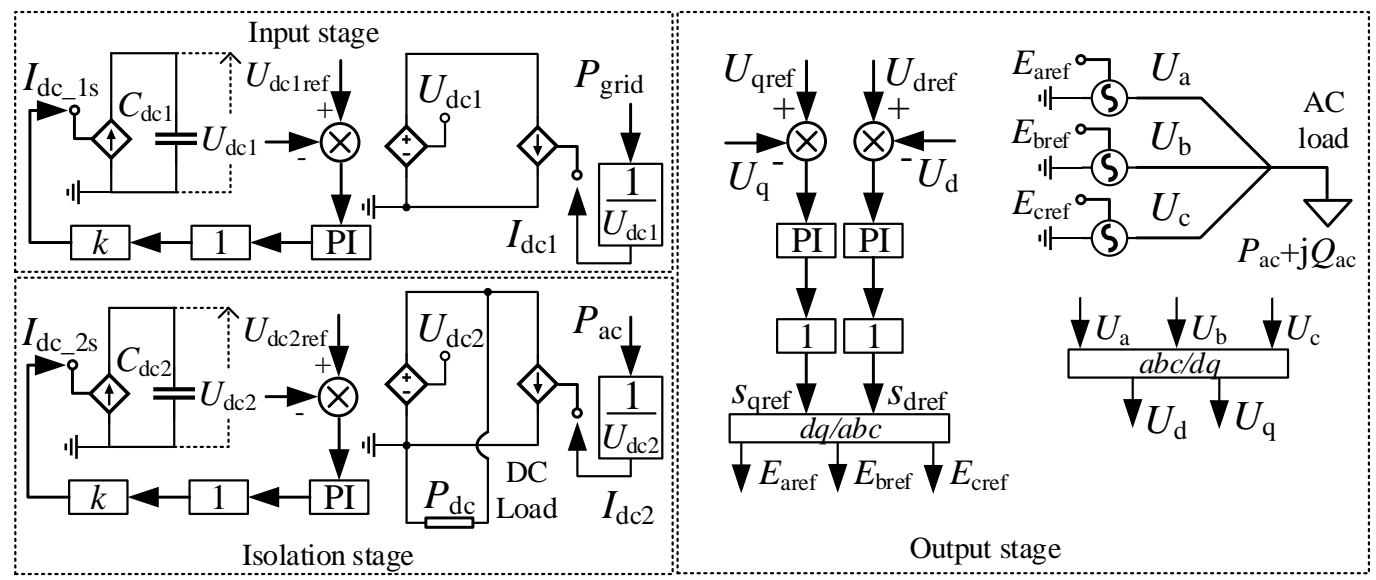

Figure 6. Simplified model structure of the microgrid.

The input stage is simplified to a controlled voltage source. The voltage reference signal $U_{\mathrm{dc} 1 \mathrm{ref}}$ is compared with the capacitor voltage $U_{\mathrm{dc} 1}$, the error of which is processed by the PI regulator to output a current inner loop reference signal. Since the current inner loop is simplified, the actual value of the inner loop current signal is considered to be equal to the reference value, which multiplies the proportional coefficient $k$ to generate the controlled current source reference signal $I_{\mathrm{dc} \_1 \mathrm{~s}}$, so that the capacitor voltage is stabilized at the reference value. The current signal $I_{\mathrm{dc} 1}$ from the DC terminal to the isolation stage is obtained from dividing the grid power signal by the voltage signal. The isolation stage simplification method is similar to that of the input stage, which is simplified to a controlled voltage source supplying for a DC load. The output stage is simplified to a three-phase controlled voltage source. The voltage outer loop reference signals $U_{\mathrm{dref}}$ and $U_{\mathrm{qref}}$ are compared at actual values $U_{\mathrm{d}}$ and $U_{\mathrm{q}}$, respectively, the errors of which are processed by the PI (proportional-integral) regulator to generate the output current inner loop reference signal. The inner loop output value, which gets the coordinate inverse, transforms to obtain the reference signal of the three-phase controlled voltage source and is equivalent to the input reference signal value. The stages are connected by the power balance relationship.

\section{Results}

\subsection{Simulation Time and Accuracy Analysis}

In order to verify the correctness of the simplified model, the micro-grid electromagnetic transient detailed model and simplified model were built on the PSCAD software platform to analyze. The input stage was connected to a $10 \mathrm{kV} / 50 \mathrm{~Hz}$ AC grid, which is considered an infinite system with a constant output voltage. The DC voltage on the input stage side was $18 \mathrm{kV}$. The frequency of the high frequency square wave of the isolation stage was $10 \mathrm{kHz}$, where the high frequency transformer transformation ratio was $18 \mathrm{kV}: 0.75 \mathrm{kV}$. The DC voltage on the output stage side was $0.75 \mathrm{kV}$, the output of which was $0.38 \mathrm{kV} / 50 \mathrm{~Hz}$ AC. The AC load was $0.09 \mathrm{MW}$ and the DC load was $0.2 \mathrm{MW}$. The simulation steps were 5 and $20 \mu \mathrm{s}$, respectively, and the simulation duration was $2 \mathrm{~s}$. The corresponding time-consumption comparison results are shown in Table 1.

Table 1. Time-consuming comparison results.

\begin{tabular}{ccccc}
\hline Type & $\begin{array}{c}\text { Simulation Step } \\
\text { Size/ } \mu \text { s }\end{array}$ & Detailed Model & Simplified Model & $\begin{array}{c}\text { Time-Consumption } \\
\text { Ratio }\end{array}$ \\
\hline \multirow{2}{*}{ Microgrid } & 5 & 398 & 39 & 10.2 \\
& 20 & 122 & 18 & 6.8 \\
\hline
\end{tabular}


As shown in Table 1, under the same simulation step conditions, since the simplified model replaces the converter with controlled sources and simplifies the control loop, the time consumption was significantly shorter than that of the switch model. The finer the simulation step size was, the more obvious the time-consumption reduction effect was.

At $\mathrm{T}=1 \mathrm{~s}$, the $0.09 \mathrm{MW}$ AC load was input, and the $0.1 \mathrm{MW}$ DC load was cut off at $1.5 \mathrm{~s}$. The dynamic characteristics and accuracy of the model are shown in Figure 7 and Table 2.
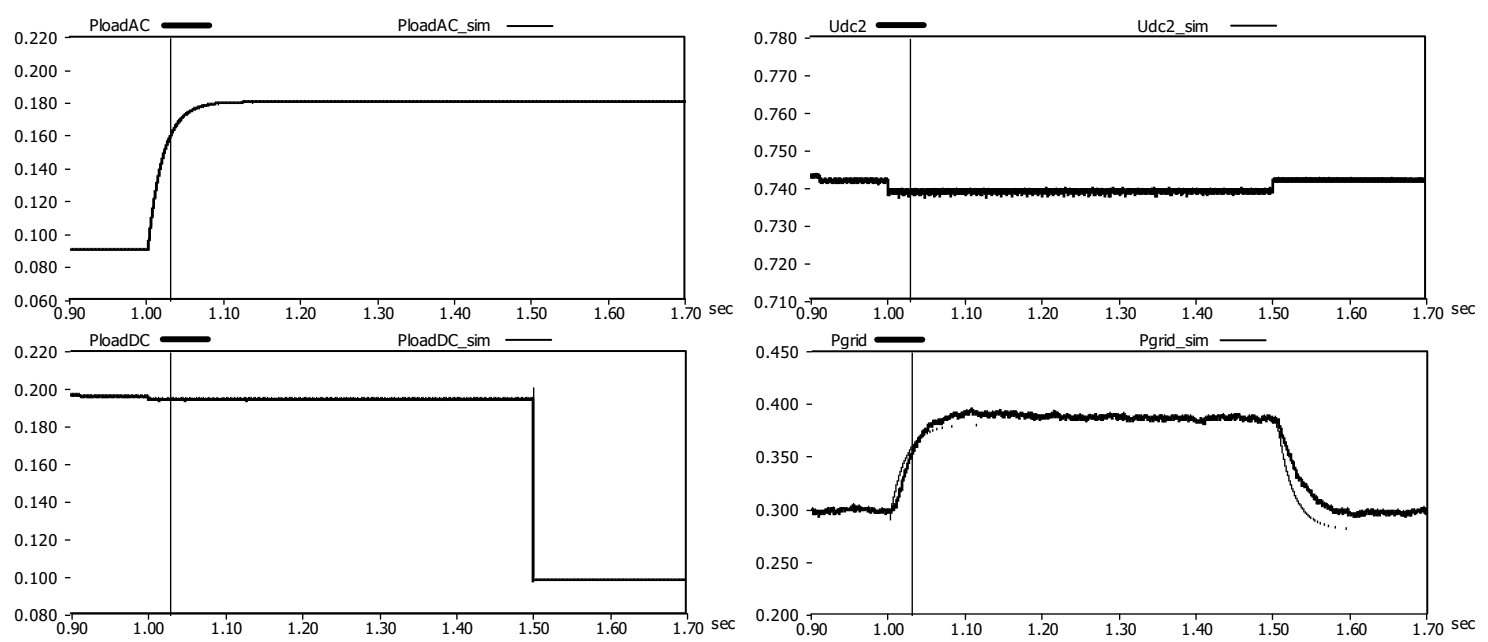

Figure 7. Comparison of output characteristics under load switching.

Table 2. Accuracy comparison results.

\begin{tabular}{ccccc}
\hline \multicolumn{2}{c}{ Output Steady State Value } & $\mathbf{0 - 1 ~ s}$ & $\mathbf{1 - 1 . 5 ~ s}$ & $\mathbf{1 . 5 - 2 ~ s}$ \\
\hline \multirow{2}{*}{$P_{\text {loadAC }}$} & Detailed model & 0.090 & 0.180 & 0.180 \\
$/ \mathrm{MW}$ & Simplified model & 0.090 & 0.180 & 0.180 \\
& Error/\% & 0.0 & 0.0 & 0.0 \\
$U_{\mathrm{dc2}}$ & Detailed model & 0.743 & 0.738 & 0.743 \\
$/ \mathrm{MW}$ & Simplified model & 0.750 & 0.750 & 0.750 \\
& Error/\% & 0.9 & 1.6 & 0.9 \\
$P_{\text {loadDC }}$ & Detailed model & 0.196 & 0.194 & 0.098 \\
$/ \mathrm{MW}$ & Simplified model & 0.200 & 0.200 & 0.100 \\
& Error/\% & 2.0 & 3.1 & 2.0 \\
$P_{\text {grid }}$ & Detailed model & 0.296 & 0.388 & 0.287 \\
$/ \mathrm{MW}$ & Simplified model & 0.290 & 0.380 & 0.280 \\
& Error/\% & 2.0 & 2.1 & 2.4 \\
\hline
\end{tabular}

As shown in Figure 7, the dynamic curve of the simplified model proposed in this paper was basically consistent with that of the detailed model. The AC load power $P_{\text {loadAC_sim }}$ curve of the simplified model and the AC load power $P_{\text {loadAC }}$ curve of the detailed model were almost identical, both of which increased due to the input of the AC load. The DC voltage $U_{\mathrm{dc} 2}$ of the detailed model fluctuated slightly due to the switching of the load, and the DC voltage $U_{\mathrm{dc} 2 \_s i m}$ of the simplified model was always constant because the reference signal remained constant. At $\mathrm{T}=1 \mathrm{~s}$, when the AC load was input, the DC load power $P_{\text {loadDC }}$ of the detailed model also had a small fluctuation due to the small fluctuation of the DC voltage $U_{\mathrm{dc} 2}$. Since the DC voltage $U_{\mathrm{dc} 2 \text { sim }}$ of the simplified model remained stable, the DC load power $P_{\text {loadDC_sim }}$ of the simplified model also remained stable. At $\mathrm{T}=1.5 \mathrm{~s}$, due to the cut off of the DC load, the DC load power of both the detailed model and the simplified model decreased. At $\mathrm{T}=1 \mathrm{~s}$, due to the input of AC load, the grid power increased. At $\mathrm{T}=1.5 \mathrm{~s}$, the DC load was cut off, and the grid power decreased.

Since the simplified model replaces the converter with controlled sources, there was no loss of the grid power, but there were slight errors. The data in Table 2 show that the output steady state 
errors between the simplified model and detailed model were within $5 \%$, which meets the requirement of engineering calculation precision [21].

\subsection{Characteristic Analysis under Fault Conditions}

In order to verify the effectiveness of the simplified model under fault conditions and to observe its dynamic characteristics under fault conditions, the action of the protection system was not considered, and the load did not change.

\subsubsection{Characteristic Analysis under AC Load Side Fault Conditions}

A single-phase ground fault occurred on the AC load side of the microgrid system at $1 \mathrm{~s}$. The dynamic characteristics are shown in Figure 8.
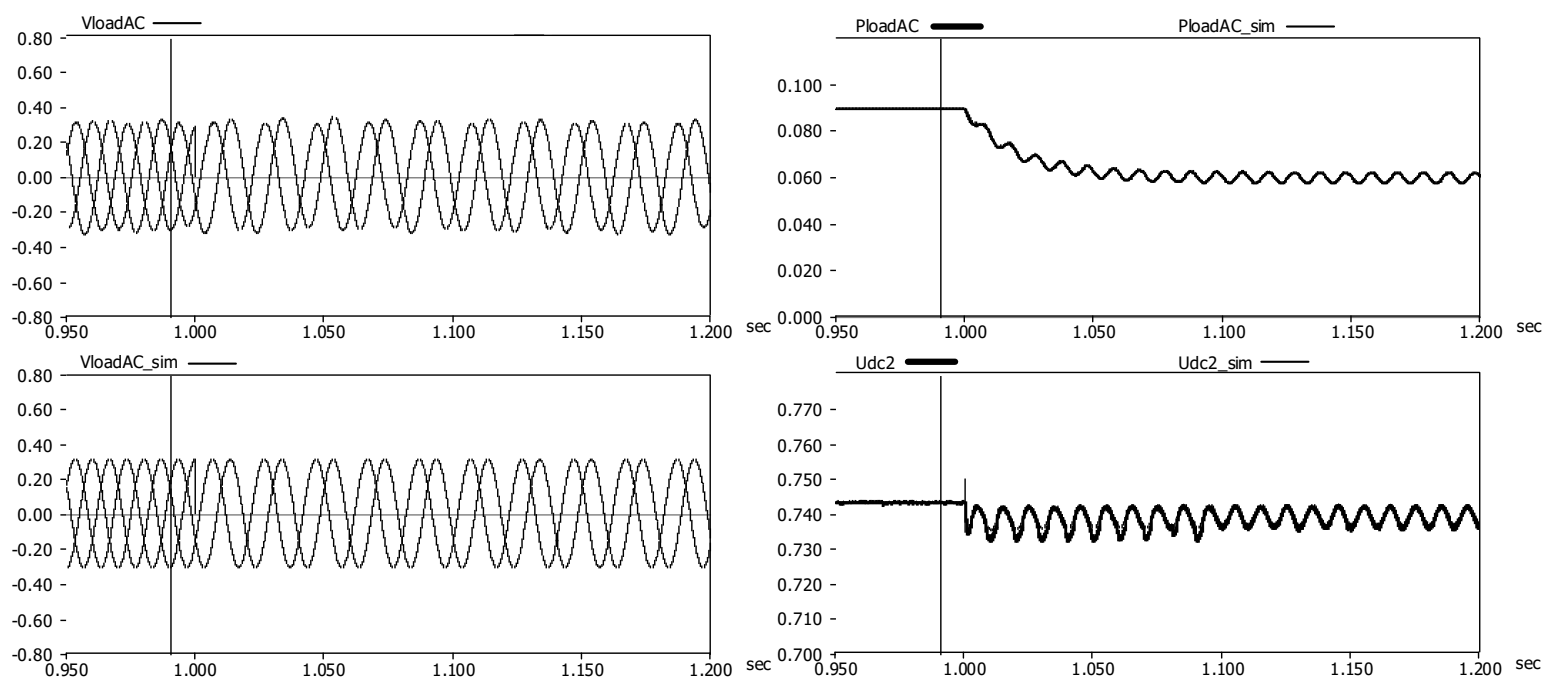

Figure 8. Output characteristics under AC load side fault conditions.

As shown in Figure 8, at $1 \mathrm{~s}$, the a-phase voltage dropped to 0 due to the a-phase failure, and the $\mathrm{b}$ and c-phase voltages remained stable. The AC load voltage $V_{\text {loadAC_sim }}$ curve of the simplified model was able to maintain good consistency with that ( $\left.V_{\text {loadAC }}\right)$ of the detailed model.

When a three-phase asymmetric fault occurs on the AC side, the output power of the AC side contains a second-harmonic disturbance [22], as shown in Equation (2).

$$
\left\{\begin{array}{c}
P_{\mathrm{ac}}=P_{0}+P_{\mathrm{c} 2} \cos (2 \omega t)+P_{\mathrm{s} 2} \sin (2 \omega t) \\
Q_{\mathrm{ac}}=Q_{0}+Q_{\mathrm{c} 2} \cos (2 \omega t)+Q_{\mathrm{s} 2} \sin (2 \omega t)
\end{array}\right.
$$

$P_{0}, P_{\mathrm{c} 2}, P_{\mathrm{s} 2}, Q_{0}, Q_{\mathrm{c} 2}$, and $Q_{\mathrm{s} 2}$ are AC power coefficients, which are related to voltage and current in a positive and negative sequence synchronous rotation coordinate system [14]. According to the principle of conservation of energy, there is also a second-harmonic disturbance equal to it on the DC side power, as shown in Equation (3).

$$
P_{\mathrm{dc} 2}=P_{\mathrm{ac}}=P_{\mathrm{dc}^{\prime}}+P_{\mathrm{c}}
$$

where, $P_{\mathrm{dc} 2}$ is the DC power received by the output stage; $P_{\mathrm{ac}}$ is the AC power flowing through the output stage; $P_{\mathrm{dc} 2}$ is the output DC power of the isolation stage; and $P_{\mathrm{ac}}$ is the capacitor discharging power.

The capacitor discharging power is as shown in Equation (4):

$$
\frac{1}{2} C \cdot u_{\mathrm{c}} \cdot \frac{d u_{\mathrm{c}}}{d t}=P_{0}+P_{\mathrm{c} 2} \cos (2 \omega t)+P_{\mathrm{s} 2} \sin (2 \omega t)-P_{\mathrm{dc} 2^{\prime}}
$$


After the fault occurred, the AC load power dropped to around $0.06 \mathrm{MW}$ containing a second-harmonic disturbance. The dynamic curves of the AC load power $P_{\text {loadAC_sim }}$ of the simplified model were almost the same as those $\left(P_{\text {loadAC }}\right)$ of the detailed model. The DC load voltage $U_{\mathrm{dc} 2}$ also contained a second-harmonic disturbance. The DC load voltage curves of the two models $\left(U_{\mathrm{dc} 2}\right.$ and $\left.U_{\mathrm{dc2} \_s i m}\right)$ were also consistent with a very slight error. In conclusion, the dynamic characteristics of the simplified model maintain good consistency with those of the detailed model.

\subsubsection{Characteristic Analysis under DC Load Side Fault Conditions}

A two-pole short circuit fault occurred on the DC load side of the microgrid system at $1 \mathrm{~s}$. The dynamic characteristics are shown in Figure 9.
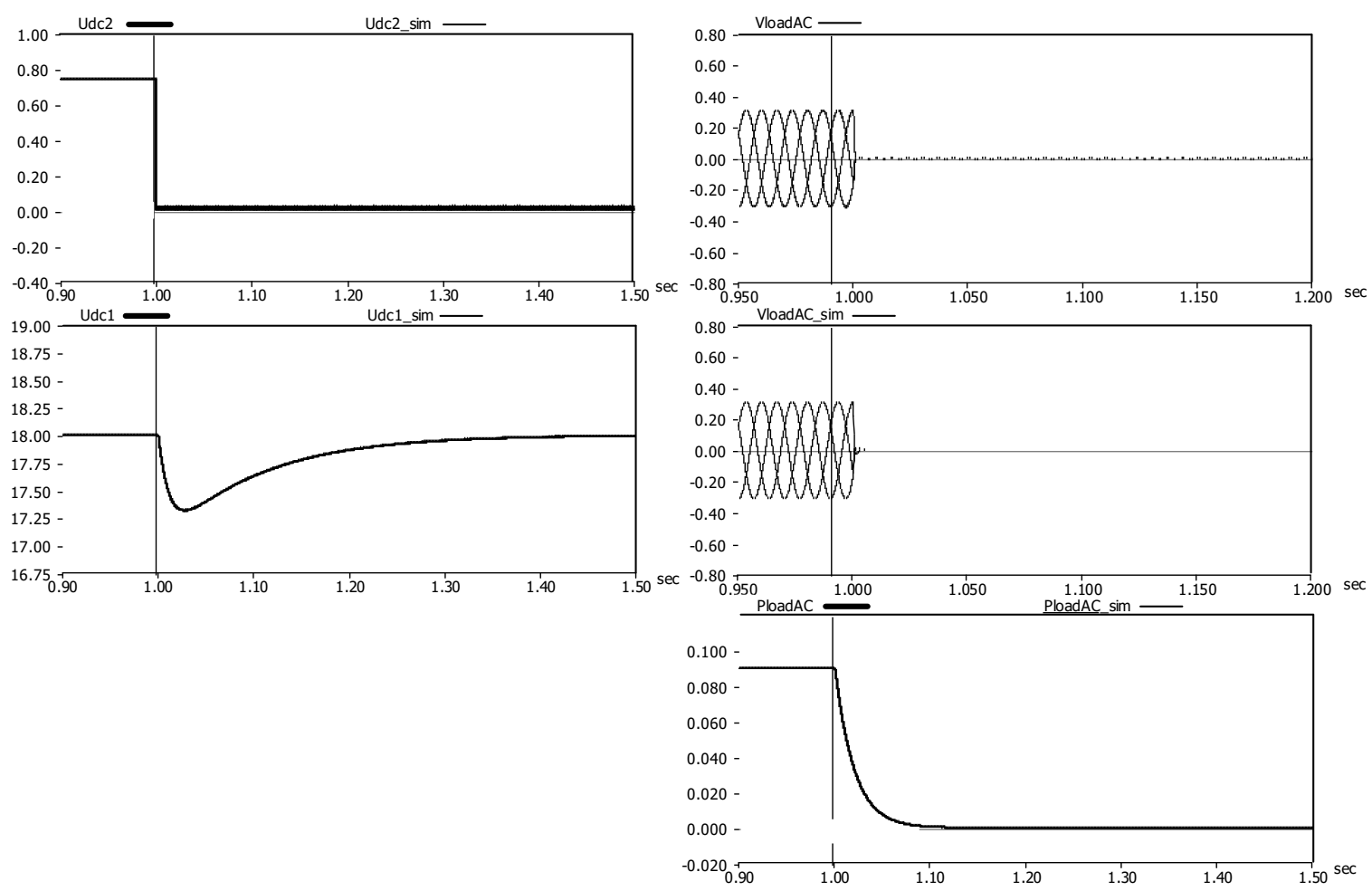

Figure 9. Output characteristics under DC load side fault conditions.

When a two-stage short circuit fault occurs on the DC side due to the DC voltage dropping to zero and the freewheeling effect of the short-circuit reactance, it is equivalent to a three-phase short circuit occurring on the AC side [10]. Its equivalent circuit is shown in Figure 10.

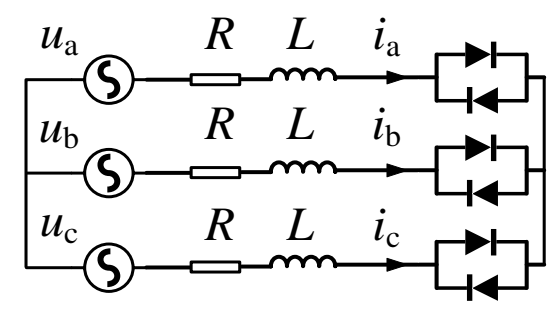

Figure 10. AC side equivalent circuit after a two-pole short circuit fault occurred.

As shown in Figure 9, when a two-pole short-circuit fault occurred on the DC load side at $1 \mathrm{~s}$, the DC load voltage $U_{\mathrm{dc} 2}$ suddenly dropped, which also caused the fluctuation of the input-stage side DC voltage $U_{\mathrm{d} 1}$. The DC load voltage $U_{\mathrm{dc} 2}$ waveform of the simplified model and that $\left(U_{\mathrm{dc} 2 \_s i m}\right)$ 
of the detailed model almost overlapped. Although the waveform of $U_{\mathrm{d} 1}$ in the detailed model fluctuated, its steady-state value was consistent with that $\left(U_{\mathrm{d} 1 \_s i m}\right)$ of the simplified model. The AC load voltage $V_{\text {loadAC_sim }}$ curve of the simplified model maintained good consistency with that $\left(V_{\text {loadAC }}\right)$ of the detailed model. As shown in Figure 10, the three-phase AC voltage $V_{\text {loadAC }}$ of the output stage dropped to zero due to an equivalent three-phase short circuit, and the AC load power $P_{\text {loadAC }}$ also dropped to zero. This shows that the dynamic characteristics of the simplified model maintain good consistency with that of the detailed model.

\section{Conclusions}

In the view of the low efficiency of electromagnetic simulation of the AC/DC hybrid microgrid, this paper proposes a simplified AC/DC hybrid model. Following the model simulation verification of this paper, the following conclusions can be made:

1. In the AC/DC hybrid microgrid model, the microgrid network, except for the generators, was taken as the research object. This model transforms the response time scale to milliseconds, simplifies the converter and its control system, and applies controllable power sources to replace the converter.

2. Through the mathematical model equivalent simplification method, there is no need to apply a new program or algorithm. The reduction of the amount of simulation data can reduce the dependence on the performance of the computer.

3. The simplified model proposed in this paper improves the simulation efficiency, the output characteristics of which maintain good consistency with those of the detailed model under AC or DC load side fault conditions.

4. The simplification of this paper is for each rectifier or inverter module connected by the power balance relationship. It could be applied to other more complex network topologies including more modules.

Author Contributions: The authors contribute as follows: conceptualization, T.Y.; methodology, G.Z., T.Y.; software, Z.L. (Zhiwei Li); validation, T.Y., J.Q.; formal analysis, Z.L. (Zhiwei Li); investigation and resources, T.Y., G.Z., Z.L. (Zhaoxiong Li); data curation, Q.Z.; writing—original draft preparation, G.Z., Z.L. (Zhaoxiong Li); writing-review and editing, T.Y.; supervision, G.Z.; project administration, T.Y. All authors have read and agreed to the published version of the manuscript.

Funding: This research received no external funding.

Conflicts of Interest: The authors declare no conflict of interest.

\section{References}

1. Zhao, G.; Wang, D. Comprehensive evaluation of AC/DC hybrid microgrid planning based on analytic hierarchy process and entropy weight method. Appl. Sci. 2019, 9, 3843. [CrossRef]

2. Pan, H.; Ding, M.; Chen, A.; Bi, R.; Sun, L.; Shi, S. Research on distributed power capacity and site optimization planning of AC/DC hybrid micrograms considering line factors. Energies 2018, 11, 1930. [CrossRef]

3. Gai, X.; Wang, Y.; Chen, R.; Zou, L. Research on hybrid microgrid based on simultaneous AC and DC distribution network and its power router. Energies 2019, 12, 1077. [CrossRef]

4. Asghar, F.; Talha, M.; Kim, S.H. Robust frequency and voltage stability control strategy for standalone AC/DC hybrid microgrid. Energies 2017, 10, 760. [CrossRef]

5. Baek, J.; Choi, W.; Chae, S. Distributed control strategy for autonomous operation of hybrid AC/DC microgrid. Energies 2017, 10, 373. [CrossRef]

6. Brando, G.; Bova, B.; Cervone, A.; Dannier, A.; Del Pizzo, A. A distribution power electronic transformer with MMC. Appl. Sci. 2018, 8, 120. [CrossRef]

7. Shi, S.M.; Wang, X.F.; Zhong, F. The research of typical control strategy of electronic power transformer in parallel operation. In Proceedings of the IEEE Advanced Information Management, Communicates, Electronic and Automation Control Conference (IMCEC), Xi'an, China, 3-5 October 2016. 
8. Duan, Q.; Wang, J.H.; Ma, C.Y. Flexible power distribution unit-A novel power electronic transformer development and demonstration for distribution system. In Proceedings of the IECON 41st Annual Conference of the IEEE Industrial Electronics Society, Yokohama, Japan, 9-12 November 2015.

9. Zhu, M.L.; Geng, S.B.; Li, Y.G. Protected control strategies for voltage-source-converter (VSC) under grid faults in AC/DC hybrid grid. In Proceedings of the International Power Electronics and Application Conference and Exposition, Shanghai, China, 5-8 November 2014.

10. Yang, J.; John, E.F.; John, O. Short-circuit and ground fault analyses and location in VSC-based DC network cables. IEEE Trans. Ind. Electron. 2012, 59, 3827-3837. [CrossRef]

11. Dolara, A.; Ogliari, E.; Raboni, P. Ground fault analysis in a microgrid scenario. In Proceedings of the IEEE Milan PowerTech, Milan, Italy, 23-27 June 2019.

12. Ramazan, B.; Erdal, I.; Fatih, I. Short-circuit fault analysis on microgrid. In Proceedings of the International Conference on Renewable Energy Research and Applications (ICRERA), Palermo, Italy, 22-25 November 2015.

13. Zhao, M.; Zheng, X. Waveform characteristic analysis and recognition of short-circuit fault in grid-connected AC microgrid. In Proceedings of the 4th International Conference on Intelligent Green Building and Smart Grid (IGBSG), Yichang, China, 6-9 September 2019.

14. Wang, Z.; Li, Y.; Li, Z.; Zhao, C.; Gao, F.; Wang, P. Reduced-order DC terminal dynamic model for multi-port AC-DC power electronic transformer. Energies 2019, 12, 2130. [CrossRef]

15. Md, R.; Jacob, A.M.; Jonathan, W.K. Reduced-order small-signal model of microgrid systems. IEEE Trans. Sustain. Energy 2015, 6, 1292-1305.

16. Petr, V.; Huang, P.H.; Mohamed, A.H. High-fidelity model order reduction for microgrids stability assessment. IEEE Trans. Power Syst. 2018, 33, 874-887.

17. Valerio, M.; Francesco, V.; Juan, C.V. Model order reductions for stability analysis of islanded microgrids with droop control. IEEE Trans. Ind. Electron. 2015, 62, 4344-4354.

18. Peng, Y.L.; Shuai, Z.K.; John, S. Reduced order modeling method of inverter-based microgrid for stability analysis. In Proceedings of the IEEE Applied Power Electronics Conference and Exposition (APEC), Tampa, FL, USA, 26-30 March 2017.

19. Wang, Y.; Lu, Z.X.; Min, Y. Small signal analysis of microgrid with multiple micro sources based on reduced order model in islanding operation. In Proceedings of the IEEE Power and Energy Society General Meeting, Detroit, MI, USA, 24-28 July 2011.

20. Alsseid, A.M.; Jovcic, D.; Starkey, A. Small signal modelling and stability analysis of multiterminal VSC-HVDC. In Proceedings of the 14th European Conference on Power Electronics and Applications, Birmingham, UK, 30 August-1 September 2011; pp. 1-10.

21. Liu, X.J.; Guo, D. Study on equivalent simplified model of photovoltaic power generation system. Acta Energy Sol. Sin. 2016, 37, 759-764.

22. Xu, K.; Wang, W.J.; Jia, L.H. Influence of AC unbalanced fault on DC side of hybrid AC/DC microgrid. Proc. CSEE 2018, 38, 4429-4437.

(C) 2020 by the authors. Licensee MDPI, Basel, Switzerland. This article is an open access article distributed under the terms and conditions of the Creative Commons Attribution (CC BY) license (http://creativecommons.org/licenses/by/4.0/). 